\title{
Raum und Verkehr - ein Feld komplexer Wirkungsbeziehungen. Können Interventionen in die gebaute Umwelt klimawirksame Verkehrsemissionen wirklich senken?
}

\author{
Christian Holz-Rau' ${ }^{1}$ Joachim Scheiner ${ }^{1}$
}

Eingegangen: 22. Dezember 2015 / Angenommen: 20. Juli 2016 / Online publiziert: 3. August 2016

(c) Springer-Verlag Berlin Heidelberg 2016

Zusammenfassung Mit dem Bild einer integrierten Standort- und Verkehrsplanung verknüpft sich die Hoffnung, die bisher verkehrsinduzierend wirkenden Wechselwirkungen zwischen Raum und Verkehr quasi umzudrehen und durch planerische Interventionen in den Bereichen von Standortstruktur und Verkehrsangebot einen nennenswerten Beitrag zur Reduzierung der klimarelevanten Emissionen zu leisten. Die planerische Gestaltung nutzungsgemischter und kompakter Standortstrukturen auf kommunaler und regionaler Ebene wird allerdings überlagert von gesellschaftlichen und räumlichen Entwicklungen, für die großräumige Mobilität politisch erwünscht oder strukturell notwendig ist. Vor diesem Hintergrund erscheinen die genannten Hoffnungen deutlich übertrieben. Wir stellen in diesem Beitrag bekannte empirische Ergebnisse zu Raumstrukturen und Verkehr in einen anderen Interpretationszusammenhang und lenken den Blick auf unseres Erachtens wichtigere Treiber der Verkehrsentwicklung, die allerdings außerhalb des Handlungsfeldes einer integrierten Standort- und Verkehrsplanung stehen. Wir ziehen die Schlussfolgerung, dass eine integrierte Standort- und Verkehrsplanung nicht mit der Vermeidung von $\mathrm{CO}_{2}$-Emissionen begründet werden sollte. Trotzdem bleibt sie aus unserer Sicht sinnvoll, da eine integrierte Standort- und Verkehrsplanung zu weiteren Zielen der Stadtentwicklung beitragen kann.

Prof. Dr. Christian Holz-Rau

christian.holz-rau@tu-dortmund.de

Prof. Dr. Joachim Scheiner

joachim.scheiner@tu-dortmund.de

1 Fachgebiet Verkehrswesen und Verkehrsplanung,

Technische Universität Dortmund,

August-Schmidt-Straße 10, 44227 Dortmund,

Deutschland
Schlüsselwörter Mobilität · Verkehr · Raumentwicklung · Reurbanisierung $\cdot$ Klimaschutz $\cdot$ Verkehrsplanung

Space and Traffic - A Field of Complex Interrelations. Can Interventions in the Built Environment Really Reduce Transport-Related Climate Emissions?

Abstract The image of an integrated urban and transport planning is linked to the hope for a turnaround in the mutual interrelations between the built environment and transport that have actually induced more transport to date. Planning interventions in location structures and transport supply should therefore effectively contribute to reduce transportrelated climate emissions. However, the targeted design of mixed land-use and compact location structures on the local and regional level is superimposed by societal and spatial trends that make large-scale mobility politically desirable or necessary. Against this background, the hopes mentioned before appear widely overstated. In this paper we put wellknown empirical findings in new contexts of interpretation, and we point to other - from our perspective, more important - drivers of transport trends that are beyond the scope of integrated urban and transport planning. We conclude that integrated urban and transport planning should not be justified by avoiding carbon dioxide emissions, but remains reasonable for other reasons.

Keywords Mobility - Traffic - Spatial development . Reurbanisation $\cdot$ Climate protection $\cdot$ Traffic planning 


\section{Einleitung}

Die Verkehrsentwicklung in Deutschland und Europa ist seit dem Zweiten Weltkrieg durch eine starke Zunahme des Personen- und Güterverkehrs, vor allem mit Pkw und Lkw, gekennzeichnet. Im Personenverkehr in Deutschland haben sich die zurückgelegten Distanzen von $9 \mathrm{~km}$ (1950) auf $40 \mathrm{~km}$ pro Person und Tag (2013) vervierfacht, die Distanzen mit dem motorisierten Individualverkehr dabei von 1,8 auf $31 \mathrm{~km}$ versiebzehnfacht. Der Lkw-Verkehr hat noch stärker zugenommen, nämlich von 286 auf 5519 Tonnenkilometer je Einwohner, das heißt um den Faktor 19 (BMVI 2014, eigene Berechnungen). Diese Verkehrszunahme verlief in einem komplexen Prozess gesellschaftlicher und raumstruktureller Veränderungen.

Der Begriff der „Wechselwirkungen zwischen Raum und Verkehr" spielt dabei im Planungsdiskurs eine große Rolle. Gemeint sind hiermit zum einen sinkende Raumwiderstände (vor allem durch Infrastrukturausbau und zunehmender Pkw-Bestand) als Treiber disperser und entmischter Siedlungsentwicklung in geringer Dichte und zum anderen diese Siedlungsentwicklung wiederum als Treiber des Infrastrukturausbaus, steigender Motorisierung und Zunahme der zurückgelegten Distanzen.

Umgekehrt verknüpft sich mit einer integrierten Standort- und Verkehrsplanung die Hoffnung, diese bisher verkehrsinduzierend wirkenden Wechselwirkungen (Cervero 2003) quasi umdrehen zu können, die zurückgelegten Distanzen durch planerische Interventionen in den Bereichen von Raumstruktur und Verkehrsangebot zu verkürzen (Verkehrsvermeidung) und den Verkehr vom Pkw auf umweltfreundlichere Verkehrsmittel zu verlagern (Verkehrsverlagerung). Diese Diskussion blendet jedoch besonders klimarelevante gesellschaftliche Treiber der Verkehrsentwicklung aus (z. B. Wirtschaftswachstum, Globalisierung, Bildungsexpansion, Emanzipation). Die absehbaren gesellschaftlichen Entwicklungen lassen einen weiteren Anstieg der zurückgelegten Distanzen und eine Zunahme besonders weiter Wege erwarten (vom Fernpendeln bis zur interkontinentalen Privat- oder Geschäftsreise). Diese liegen bisher weitgehend außerhalb der (wissenschaftlichen und planerischen) Betrachtung.

Vor dem Hintergrund dieser gesellschaftlichen Trends und des damit verbundenen Verkehrswachstums erscheinen die Hoffnungen auf eine nennenswerte Reduktion klimarelevanter Emissionen im Verkehr durch raumstrukturelle Konzepte übertrieben. Daher setzen wir uns in diesem Beitrag vor allem mit drei Fragen auseinander:

1. Sind die Veränderungen von Raum- und Verkehrsstrukturen vorrangig im Sinne der Wechselwirkungen gegenseitig oder durch andere, möglicherweise wichtigere Treiber bedingt?
2. Sind entsprechende Interventionen, wenn sie denn wirksam sein sollten, überhaupt umsetzbar?

3. Lassen sich in dieser Folge durch eine abgestimmte Planung von Standortstrukturen und Verkehrsangeboten die Verkehrsstrukturen so beeinflussen, dass die klimarelevanten Emissionen des Verkehrs nennenswert abnehmen?

Der Beitrag berichtet keine originäre, neue Empirie, sondern ist eine auf theoretische und empirische Literatur gestützte Positionsbestimmung. Wir argumentieren dabei wie folgt. Kap. 2 stellt eine in der Planungsdiskussion weit verbreitete Sichtweise dar, die das oben beschriebene Verständnis der Wechselwirkungen zwischen Raum- und Verkehrsstruktur in den Mittelpunkt stellt. In diesem Kontext werden unter Raumstruktur in der Regel die räumliche Verteilung und Dichte von Flächennutzungen verstanden, die im Personenverkehr als Quellen und Ziele von Wegen fungieren. Wir verwenden hierfür im Weiteren den Begriff ,gebaute Umwelt“" (vgl. Ewing/Cervero 2010; Cao 2014), um ihn gegenüber anderen (z. B. sozialen und konstruktivistischen) Raumbegriffen abzugrenzen (vgl. Hard 2008). Gleichzeitig sprechen wir von einer integrierten Standort- und Verkehrsplanung. Diese reicht von der Ausweisung von Flächennutzungen und Festlegung von Dichten über die Gestaltung der Verkehrsinfrastruktur bis zum Mobilitätsmanagement. Gemeinsam sind Standort- und Verkehrsplanung als wesentliche Bestandteile einer übergreifenden Raumplanung. Die Aussagen beziehen sich entsprechend der kommunalen, teilweise auch regionalen Zuständigkeiten für die Standortplanung vor allem auf diese räumlichen Ebenen. Dabei sei ergänzt, dass nicht die Planung und der politische Beschluss selbst (verkehrs-)wirksam sein können, sondern erst deren Realisierung, die im Übrigen häufig mehr oder weniger stark von der ursprünglichen Planung abweicht.

Kap. 3 stellt kurz empirische Befunde zum Zusammenhang zwischen der gebauten Umwelt und dem Verkehrsverhalten in dieser dar. Anhand von Vergleichen des Verkehrsverhaltens der Wohnbevölkerung werden ,verkehrssparsame“ und „verkehrsaufwendige“ Teilräume identifiziert. Dabei werden allerdings Wechselwirkungen weitgehend ausgeblendet, da es sich in der Regel um zeitliche Momentaufnahmen handelt. Problematisch ist für die Planungsdiskussion, dass die beobachteten Unterschiede zwischen Teilräumen als Verkehrssparpotenziale angesehen werden und (schwer durchschaubare) Verkehrsprognosemodelle diese Unterschiede zugrunde legen, wenn sie die Verkehrsfolgen verschiedener räumlicher Szenarien abbilden sollen.

Deshalb stellen wir in Kap. 4 übliche planerische Schlussfolgerungen der in Kap. 3 dargestellten Befunde in Frage, und zwar im Hinblick auf die (fehlende) Kausalität und fehlende zeitliche Stabilität der untersuchten 
Zusammenhänge sowie hinsichtlich der (fehlenden) Gestaltbarkeit der Ursachen.

Das Konzept der Wechselwirkungen zwischen Raum und Verkehr ist empirisch gut fundiert, blendet aber andere, aus unserer Sicht dominante gesellschaftliche Veränderungen aus, die ihrerseits die Raum- und Verkehrsentwicklung prägen. Diese führen wir exemplarisch in Kap. 5 aus. Raumund Verkehrsentwicklung verstehen wir hiernach als Dimensionen gesellschaftlicher Veränderungen, die sich in weiten Teilen außerhalb des Interventionsfeldes der Standort- und Verkehrsplanung vollziehen. Diese führen zu einer Ausdehnung der Aktionsräume im Alltags- und Fernverkehr von Personen. Dies gilt analog für Güternah- und Güterfernverkehre, die hier jedoch nicht betrachtet werden.

Diesen Betrachtungen zufolge überschätzt die planerische Diskussion die Wirksamkeit von Interventionen in die gebaute Umwelt deutlich. Dies relativiert die Hoffnungen, die sich an raumstrukturelle Maßnahmen und Konzepte zur Verkehrsvermeidung knüpfen. Dies ist wichtig im Sinne einer realistischen Selbsteinschätzung der Gestaltungsmacht der (vor allem kommunalen und regionalen) Planung, um nicht an falschen Hoffnungen zu scheitern. Sie sollten stattdessen von vornherein planerisches Handeln an realistischen Zielen und Erwartungen ausrichten und die jeweils zielführenden Akteure für ein planerisch-politisches Ziel oder Konzept benennen (z. B. Bund/EU versus Kommune), anstatt Erfolge von weitgehend wirkungslosen Interventionen und den damit verbundenen Akteuren zu erwarten oder zu fordern. Es sei aber betont, dass dieser Beitrag politikwissenschaftliche Perspektiven im Sinne einer Akteurs- oder Diskursanalyse ausblendet, auch wenn die Interessen, Diskurse und Durchsetzungsmacht zahlreicher Akteursgruppen zweifellos große Bedeutung in der Standort- und Verkehrsplanung und -politik besitzen.

Wir argumentieren hier primär mit dem Verkehrsaufwand (zurückgelegte Distanzen) und folglich mit der Strategie der Verkehrsvermeidung, weniger mit der Verkehrsmittelnutzung (Strategie Verkehrsverlagerung). Das bisherige Verkehrswachstum ging jedoch mit einer starken Zunahme gerade der Verkehrsmittel mit hohen Pro-Kopf- $\mathrm{CO}_{2}$ Emissionen einher. Verlagerungskonzepte können durchaus Erfolge vorweisen, sind allerdings angesichts des Gesamtmengenwachstums und der erforderlichen Reduktionen für einen wirksamen Klimaschutz marginal. Dabei ist einschränkend zu sagen, dass über die Stärke von Verlagerungswirkungen schwer Aussagen zu treffen sind, da die Mehrzahl entsprechender Studien zwar von statistischen Signifikanzen berichtet, aber nur wenige Studien systematisch auch Effektstärken untersuchen (vgl. van Wee/Handy 2016).

Die Einleitung abschließend, sei hier eine ergänzende Klärung vorgenommen. Der Beitrag bezieht sich nicht auf alle Wirkungsbereiche raumstruktureller Maßnahmen, wie z. B. Erreichbarkeitssicherung, Urbanität, finanzielle Belastungen für die öffentliche Hand, Flächensparsamkeit oder Landschaftsschutz. Der Beitrag argumentiert damit nicht gegen das Leitbild einer nutzungsgemischten Stadt und Region, macht aber deutlich, dass die Realisierung dieses Leitbildes verkehrsseitig keinen wesentlichen Beitrag zum Klimaschutz leisten kann. Zum Klimaschutz sind zwingend andere Konzepte erforderlich. Vor diesem Hintergrund ergeben sich abschließend Fragen für die Forschung und an dieser Stelle nur sehr knappe Anstöße für die Planung (Kap. 6).

\section{Wechselwirkungen zwischen Raum und Verkehr}

Stadtentwicklung und Verkehr waren seit jeher eng verbunden. So arbeitete etwa Lehner (1963) heraus, dass das Wachstum Berlins parallel zur Verbesserung der Verkehrsangebote verlief. Der städtische Radius entsprach jeweils etwa der Entfernung, die die allgemein verfügbaren Verkehrsmittel in einer Stunde überwinden konnten. Dabei wurde dieser Zusammenhang nicht nur darauf zurückgeführt, dass eine Verbesserung der Verkehrsangebote zum Stadtwachstum führt. Umgekehrt regt ein Wachstumsdruck auch Verbesserungen der Verkehrsangebote an.

Mit der Verbreitung des privaten Pkw begriffen viele Neu-Motorisierte das Auto als eine Möglichkeit, der Enge der Stadt zu entfliehen. Die Stadt- und Verkehrsplanung begleitete und unterstützte unter dem Leitbild von Licht, Luft und Sonne (Charta von Athen) über lange Zeit die Zunahme des Pkw- und Lkw-Verkehrs durch den Ausbau der Straßennetze und durch die Entwicklung von Neubaugebieten geringerer Dichte an den Rändern der Städte bzw. im Umland. Das Auto erleichterte die Randwanderung aus der Stadt und belastete gleichzeitig die Wohn- und Lebensverhältnisse in der Stadt. Dazu beschreibt Kutter (1975: 69 f.) einen Regelkreis, in dem erhöhte Motorisierung und steigende Mobilität über erreichbarkeitseinschränkende räumliche Veränderungen zu einer weiteren Erhöhung der Motorisierung führen und kritisiert, Mobilität und Erreichbarkeit werden ,insbesondere durch die Realisation verfehlter Leitbilder in der Stadtplanung laufend eingeschränkt" (Kutter 1975: 70). Heinze (1979) spricht unter dem Titel „Verkehr schafft Verkehr" von einem Prozess der Selbstinduktion. Ein differenziertes Modell dieses Prozesses und dessen Einbettung in die Standort- und Verkehrsplanung formuliert Wegener (2009) als Regelkreis von Stadtentwicklung und Verkehr (vgl. Abb. 1). Er betont kritisch die Wachstumsimpulse, die dabei von sinkenden Raumwiderständen (Ausbau der Verkehrsinfrastruktur, sinkende Verkehrskosten) ausgehen.

Die Basis hierfür bildet die Beobachtung einer Konstanz des Reisezeitbudgets im Personenverkehr: Die im Alltagsverkehr der entwickelten Länder aufgebrachte Zeit ist langfristig relativ konstant (Metz 2004). Sie beträgt im Bevöl- 


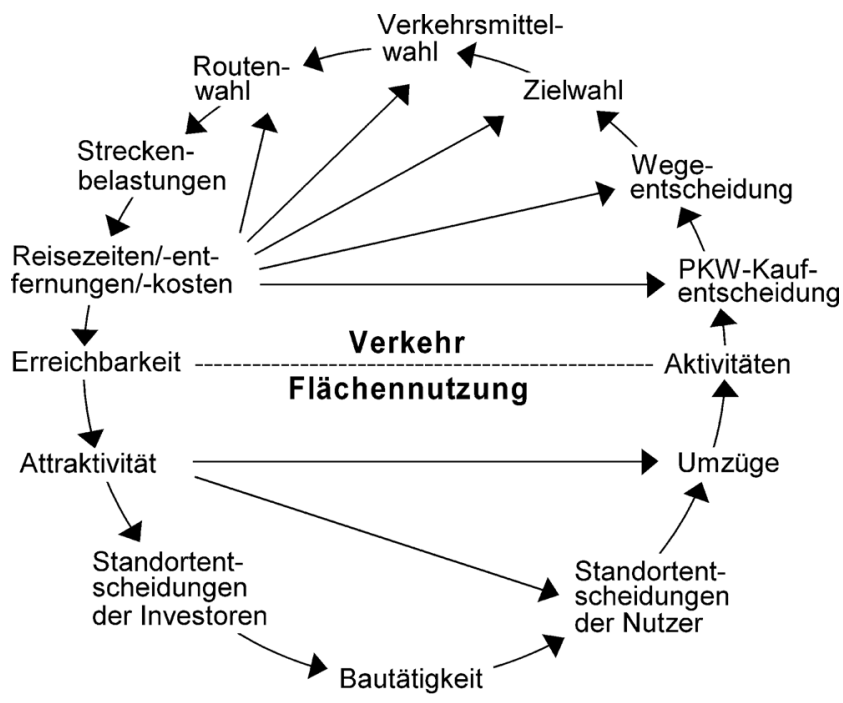

Abb. 1 Regelkreis von Verkehr und Flächennutzung (Wegener 2009: 74)

kerungsdurchschnitt etwas mehr als eine Stunde pro Person und Tag. Dabei hat seit den ersten empirischen Beobachtungen (Szalai 1972) durch die steigende Motorisierung, aber auch durch den Ausbau der Verkehrsinfrastruktur eine massive Beschleunigung stattgefunden. Diese wird aber nicht für eine Reisezeitersparnis genutzt, sondern für die Ausdehnung der Aktionsräume bei weitgehend konstanter (möglicherweise sogar leicht zunehmender (Mokhtarian/ Chen 2004)) Reisezeit. Dies wird auch als induzierter Verkehr bezeichnet. Die Konstanz des Reisezeitbudgets folgt einer allmählichen Anpassung. Wenn beispielsweise durch den Ausbau einer Straße eine Beschleunigung eintritt, werden die Nutzer dieser Straße deshalb nicht umziehen, um wieder eine Stunde täglich im Verkehr zu verbringen. Aber wenn ein Umzug oder der Wechsel eines Arbeitsplatzes ansteht, erfolgt bei höherer Raumdurchlässigkeit die Suche in einem größeren Raum.

Eine Beschleunigung eröffnet also auf der Nachfrageseite innerhalb eines relativ fixen Reisezeitbudgets neue Möglichkeiten zum Wohnen, zur Arbeit, bei Freizeit und Einkauf. Auf der Angebotsseite entstehen gleichzeitig Optionen der Konzentration und Dispersion, z. B. im Einzelhandel zur Ansiedlung von Großeinrichtungen an nichtintegrierten Standorten, im Wohnungsbau oder bei Industrieansiedlungen im Umland. Diese Entwicklungen können allerdings für Personen ohne Pkw auch zu ErreichbarkeitseinbuBen führen (soziale Exklusion) (vgl. Hesse/Scheiner 2010; Lucas 2012). Die Konstanz des Reisezeitbudgets bildet damit im Wesentlichen die Verkehrslogik des Regelkreises von Wegener (2009).

Fasst man den Regelkreis von Wegener (2009) und von Kutter (1975) vereinfacht mit Hinblick auf die Phase der privaten Motorisierung zusammen, so besagen diese zu- treffend, dass die zunehmende Verfügbarkeit über einen privaten Pkw die räumlichen Möglichkeiten privater und betrieblicher Standortentscheidungen erweitert. Diese Potenziale werden durch private Haushalte sowie private und öffentliche Institutionen genutzt. Beides führt zu mehr Verkehr, vor allem Pkw-Verkehr. Begleitet wird dieser Prozess durch den an der steigenden Nachfrage orientierten Ausbau der Verkehrsinfrastruktur (Anpassungsplanung, predict and provide) und durch die Bereitstellung von Flächen für Wohnen, Arbeiten und andere Nutzungen an verkehrsaufwendigen, Pkw-orientierten Standorten. Diesem Prozess liegen Entscheidungen der Standort- und Verkehrsplanung bzw. -politik zugrunde - oder genauer deren häufig modifizierte Realisierungen -, die in der Regel mehrheitskonform der Motorisierung breiter Bevölkerungsgruppen folgten und für die in demokratischen Systemen nur ein relativ enger Entscheidungsspielraum besteht.

Das Modell verknüpft damit Standortplanung oder präziser deren möglicherweise noch modifizierte Realisierung mit Verkehrsplanung und Verkehrsnachfrage. Es erscheint uns für das Verständnis von Raumentwicklung und Verkehrswachstum allerdings als zu planungszentriert, indem andere gesellschaftliche Prozesse, die die Raum- und Verkehrsentwicklung prägen, unberücksichtigt bleiben. So sind beispielsweise die wirtschaftliche Entwicklung, die Spezialisierung auf dem Arbeitsmarkt und die Angleichung von Geschlechterrollen weitere Entwicklungen, die die Raumund Verkehrsentwicklung prägen, teilweise aber auch von der Raum- und Verkehrsentwicklung geprägt werden (vgl. auch Kap. 4 und 5).

\section{Raumstrukturen und Verkehrsverhalten}

Parallel zu dieser Gesamtbetrachtung der Wechselwirkungen zwischen Standortstrukturen und Verkehr vertiefen zahllose Studien das Detailwissen hierzu. Danach unterscheiden sich wesentliche Kenngrößen des Alltagsverkehrs der Wohnbevölkerung in unterschiedlichen räumlichen Kontexten deutlich voneinander (Ewing/Cervero 2010). Die grundlegenden Ergebnisse dieser Studien sind in verschiedenen Ländern weitgehend ähnlich. Einige Ergebnisse vorwiegend eigener Studien können die Zusammenhänge zwischen Raum und Verkehr anhand deutscher Ergebnisse illustrieren. Die Differenzierung nach Gemeindegrößenklassen dient dabei als grobes „Vehikel“ zur Abbildung von Unterschieden in Urbanität und Verkehrssystemen.

Die folgenden Befunde sind dabei nicht im Sinne einseitig kausaler Zusammenhänge zu verstehen. Sie werden hier zusammengefasst dargestellt und dann in den Kap. 4 und 5 in ihren komplexeren Interpretationszusammenhang gestellt. Kap. 4 hinterfragt die Kausalität der Zusammenhänge zwischen der gebauten Umwelt und dem beobachte- 
ten Verkehrsverhalten, diskutiert die Problematik von Zeitschnitten sowie die Gestaltbarkeit der Ursachen. Kap. 5 ergänzt Aspekte des sozialen Wandels, die sich in der Verkehrsentwicklung niederschlagen.

Die Wohnbevölkerung von Großstädten legt im Alltagsverkehr geringere Distanzen zurück als die Wohnbevölkerung kleinerer Gemeinden. Die Spanne reicht von $27 \mathrm{~km}$ pro Person und Tag in Millionenstädten bis zu $40 \mathrm{~km}$ pro Person und Tag in Gemeinden unter 5000 Einwohnern (berechnet nach Holz-Rau/Scheiner/Sicks 2014: 497 ff. auf der Basis der Befragung „Mobilität in Deutschland 2008“1).

Innerhalb der Großstädte nimmt die durchschnittliche Alltagsdistanz der Wohnbevölkerung von innen nach außen zu. So betrugen in einer älteren Untersuchung in Berlin die Durchschnittsdistanzen der Bevölkerung des innenstadtnächsten Quartiers $11 \mathrm{~km}$ pro Tag und eines Stadtrandquartiers 20 km pro Tag (Holz-Rau 1997: 54). Ähnliche Ergebnisse zeigten sich auch in einer Studie in Köln (HolzRau/Fromberg/Gwiasda et al. 1999). Danach weisen die Durchschnittsdistanzen der Wohnbevölkerung von Quartieren mit Nebenzentren tendenziell Nebenminima auf; die Unterschiede von innen nach außen aber dominieren.

Innerhalb der Städte ist die durchschnittliche Alltagsdistanz der Wohnbevölkerung von Quartieren differenzierter Ausstattung und/oder hoher Dichte geringer als in Quartieren mit einseitiger Nutzungsstruktur und/oder geringer Dichte (Holz-Rau/Fromberg/Gwiasda et al. 1999). Dabei ist zu beachten, dass diese Merkmale der Lage im Stadtgebiet und der Quartiersstruktur in der Regel miteinander zusammenhängen.

Gleichzeitig nutzt die Wohnbevölkerung von Gemeinde- und Quartierstypen, in denen die Alltagsdistanzen unterdurchschnittlich sind, seltener den Pkw. Sie geht häufiger zu Fuß und/oder fährt häufiger mit dem Rad und mit öffentlichen Verkehrsmitteln (Holz-Rau/Scheiner/Sicks 2014). Damit verbunden ist auch die Motorisierungsrate geringer (Konrad 2016).

Erreichbarkeitsverhältnisse auf verschiedenen Maßstabsebenen konkurrieren miteinander (Handy 1992; Næss 2011). Die Wirkung einer hervorragenden, ,verkehrsvermeidenden“, nahräumlichen Ausstattung mit Einzelhandel und Dienstleistungen wird häufig außer Kraft gesetzt durch eine ebenso hervorragende Erreichbarkeit großflächiger Angebote auf der gesamtstädtischen oder regionalen Ebene.

\section{Kritische Fragen}

In der Standort- und Verkehrsplanung werden diese Befunde vielfach ,übersetzt“ in Konzepte zur Stadtentwicklung, etwa „,neo-traditional development“, ,new urbanism“ oder „transit-oriented development“ in den USA (Boarnet 2011) oder die ,kompakte Stadt“ oder „Stadt der kurzen Wege“ in Europa bzw. Deutschland (Jenks/Burton/Williams 1997; Holz-Rau/Fromberg/Gwiasda et al. 1999; Schwanen/Dijst/ Dieleman 2004). Derartige Konzepte stehen stellvertretend für den Paradigmenwechsel von der früheren Anpassungsplanung zu einer integrierten Standort- und Verkehrsplanung, die gewissermaßen „durch die Hintertür“ der Raumstruktur das Verkehrsverhalten der Bevölkerung zu steuern versucht („Gestaltungsplanung“). Auch die in der Verkehrsplanung vielfach angewandten Verkehrsberechnungsmodelle unterstellen, dass diese Unterschiede räumlich und zeitlich übertragbar sind.

In Verbindung derartiger Befunde mit den Regelkreisen von Kutter (1975) und Wegener (2009) stellt sich aber die Frage: Lassen sich diese Kreisläufe durch Planung und Politik in die Gegenrichtung drehen, anhalten oder zumindest verlangsamen? Können Standort- und Verkehrsplanung zu einer Reduzierung der Distanzen (und zu geringerer PkwNutzung) beitragen? Lässt sich durch eine integrierte Standort- und Verkehrsplanung also Verkehr vermeiden?

Für eine in diesem Sinne verkehrsvermeidende (oder auch verkehrsverlagernde) Verkehrsplanung und Verkehrspolitik müssten mindestens folgende Bedingungen erfüllt sein:

Kausalität der Zusammenhänge: Die empirisch beobachteten Unterschiede des Verkehrsverhaltens in unterschiedlichen gebauten Umwelten müssen auf kausalen Zusammenhängen basieren. Denn ohne diese Kausalität sind Veränderungen der gebauten Umwelt nicht zielgerichtet wirksam.

Zeitliche Stabilität der Zusammenhänge: Diese kausalen Zusammenhänge müssen zeitlich stabil sein. Die Prozesshaftigkeit von Ursache-Wirkungs-Zusammenhängen bleibt bei Querschnittsanalysen unsichtbar. Denn ohne eine zeitliche Stabilität der Ursache-Wirkungs-Zusammenhänge treten die erwarteten Wirkungen in der Zukunft möglicherweise nicht ein.

Gestaltbarkeit der Ursachen: Die Ursachen der Kausalbeziehungen müssen sich zielgerichtet gestalten lassen politisch und materiell. Denn ohne eine zielgerichtete Veränderung der gebauten Umwelt lassen sich auch bei kausalen Zusammenhängen keine Wirkungen erzielen.

Einfache Überlegungen und empirische Befunde zeigen, dass alle drei Bedingungen nicht erfüllt sind. Die Ausführungen sollen dazu beitragen, geodeterministische Fehlschlüsse zu vermeiden und stattdessen komplexere Wirkungsketten verdeutlichen.

$\overline{{ }^{1} \mathrm{http} / / / \mathrm{www} . m o b i l i t a e t-i n-d e u t s c h l a n d . d e / p d f / M i D 2008}$ Abschlussbericht_I.pdf (13.07.2016). 


\subsection{Zur Kausalität der Zusammenhänge}

Die im Alltagsverkehr beobachteten Unterschiede haben vielfältige Ursachen, nicht nur die Strukturen der gebauten Umwelt. Die Bevölkerungsstruktur in unterschiedlichen räumlichen Kontexten unterscheidet sich. Die Bildungsund Einkommensniveaus in den Großstädten sind meist höher als in kleineren Gemeinden. ${ }^{2}$ Da hohe Einkommen und Bildung ihrerseits mit längeren Distanzen im Alltag verbunden sind, tragen diese auch zu den Unterschieden zwischen den räumlichen Kontexten bei. So verbleiben bei multivariaten Analysen, die soziale und räumliche Einflussgrößen gleichzeitig berücksichtigen, in der Regel geringere, aber weiterhin deutliche räumliche Unterschiede.

Diese räumlichen Unterschiede sind aber Ausdruck komplexer Wirkungsketten und nicht eines direkt kausalen $\mathrm{Zu}-$ sammenhangs zwischen der gebauten Umwelt und der Verkehrsnachfrage. Hierzu trägt unter anderem der Prozess der Selbstselektion bei, der für Wohnstandortentscheidungen in den letzten Jahren vielfach untersucht wurde (vgl. Scheiner 2009; Cao 2014 und andere Beiträge im gleichen Heft), im Grundsatz aber auch alle anderen Entscheidungen über aufgesuchte Zielorte (Arbeitsplätze, Einkaufsgelegenheiten etc.) betrifft. Danach konzentrieren sich bestimmte Lebensund Mobilitätsstile an für sie besonders attraktiven Standorten. Als Gedankenexperiment formuliert Holz-Rau (1997: 38 f.): Die Bevölkerung teilt sich in einen ÖPNV-affinen und einen ÖPNV-aversen Teil. ÖPNV-affine Personen ziehen typischerweise an Standorte mit adäquater ÖPNV-Qualität. Für ÖPNV-averse Personen spielt dagegen die ÖPNVQualität keine entscheidende Rolle (vgl. Chatman 2014 am Beispiel des Fußverkehrs). Ob sie an einen Standort mit gutem ÖPNV-Angebot ziehen, hängt von anderen Eigenschaften des Standorts ab. Wenn sie es tun, werden sie den ÖPNV trotzdem kaum nutzen. Vergleicht man das Verkehrsverhalten der Bevölkerung an Standorten guter und schlechter ÖPNV-Qualität miteinander, zeigt sich eine höhere ÖPNVNutzung an Standorten guter ÖPNV-Qualität. Würde man aber die ÖPNV-Qualität dort verbessern, wo ausschließlich ÖPNV-Averse leben, hätte eine solche Verbesserung eine wesentlich geringere Wirkung, als man aufgrund des Standortvergleichs vermuten könnte.

Die Berufswege der Großstädter sind im Durchschnitt kürzer als die Berufswege der Bevölkerung kleinerer Gemeinden (Einig/Pütz 2007: 88; Elldér 2014: 128). Neben dem Aspekt der Selbstselektion - wer ungern pendelt, bevorzugt die Stadt als Wohnort - trägt hierzu die höhere

\footnotetext{
${ }^{2}$ Die hier üblicherweise genutzten Haushaltsbefragungen haben in Deutschland einen Rücklauf von inzwischen unter $30 \%$. Damit verbunden sind Selektionseffekte. Insbesondere mittlere und höhere Schichten sind überrepräsentiert. Dies kann zu den höheren Anteilen höherer Bildungs- und Einkommensniveaus in den Großstädten beitragen.
}

Zentralität der Großstädte bei, das heißt deren Ausstattungsüberschuss. In Städten, insbesondere in Innenstädten, gibt es mehr Arbeitsplätze als wohnhafte Erwerbstätige, ist das Einzelhandelsangebot umfangreicher als für die Versorgung der ansässigen Bevölkerung notwendig etc. Umgekehrt gibt es am Stadtrand und im Umland mehr Erwerbstätige als Arbeitsplätze, ist das Einzelhandelsangebot gegenüber der Nachfrage defizitär. Hier besteht durchaus ein kausaler $\mathrm{Zu}$ sammenhang zwischen der gebauten Umwelt und der Verkehrsnachfrage (Distanz und gegebenenfalls Verkehrsmittelnutzung). Ein Ausgleich dieses Ausstattungsüberschusses aber, wie auch immer dieser realisiert werden könnte, würde im Sinne dieses kausalen Zusammenhangs nicht nur an den bisher unterausgestatteten Orten zu Distanzminderungen führen, sondern an den bisher überausgestatteten Orten gleichzeitig zu Distanzzuwächsen. Das Potenzial zur Einsparung von Verkehr wäre in jedem Fall deutlich geringer als der in Querschnittsanalysen erkennbare Unterschied. Dies gilt analog für alle Handlungen, denn auch beim Einkauf, beim Schulbesuch, beim Besuch von Kinos usw. besteht an den „,verkehrssparsamen“ Standorten ein Ausstattungsüberschuss. Es zeigt sich empirisch daran, dass Panelstudien ,tend to show much smaller effects than the vast number of cross-sectional studies, which examine differences in travel behavior between places with different land-use patterns“" (van Wee/Handy 2016: 19).

Je feiner die räumliche Differenzierung der Analysen wird, umso größer werden die Unterschiede zwischen den räumlichen Einheiten. So unterscheiden sich die Tagesdistanzen der Bevölkerung zwischen den größten und kleinsten Gemeindegrößenklassen etwa um den Faktor 1,5 (27 km vs. $40 \mathrm{~km}$ ). Innerhalb der Großstädte zeigen sich zusätzlich zwischen Innenstadt und Stadtrand nochmals Unterschiede um den Faktor 2 (Holz-Rau/Fromberg/Gwiasda et al. 1999: 40 ff.). Ein durchschnittlicher Dorfbewohner in Deutschland dürfte im Alltag also etwa die 2,5- bis 3-fache Distanz zurücklegen wie ein durchschnittlicher Innenstadtbewohner einer Großstadt. Diese Unterschiede ließen sich durch Differenzierungen nach zusätzlichen Gebietsmerkmalen weiter vergrößern. Diese immer feiner differenzierenden Analysen zerlegen aber einen als Gesamtregion funktionsfähigen Raum in nicht separat funktionsfähige Teilräume. Demnach sind (mindestens) Regionen analytisch als Ganzes zu betrachten (Boarnet 2011).

\subsection{Zur Problematik von Zeitschnitten}

Die meisten Analysen des Verkehrsverhaltens basieren auf zeitlichen Querschnitten (Boarnet 2011). Auch dies kann zu einer Fehleinschätzung räumlicher Effekte beitragen, wie sich am Beispiel der Randwanderung erläutern lässt. Die Bevölkerung kleinerer Umlandgemeinden ist im Durchschnitt weiter unterwegs als die Bevölkerung größerer Um- 
Abb. 2 Ein- und Auspendlerraten im Berufsverkehr deutscher Großstädte (alte Bundesländer) (Eigene Analysen der Volkszählungen 1970 und 1987 sowie der Statistiken der Bundesanstalt für Arbeit 1999 und 2007)

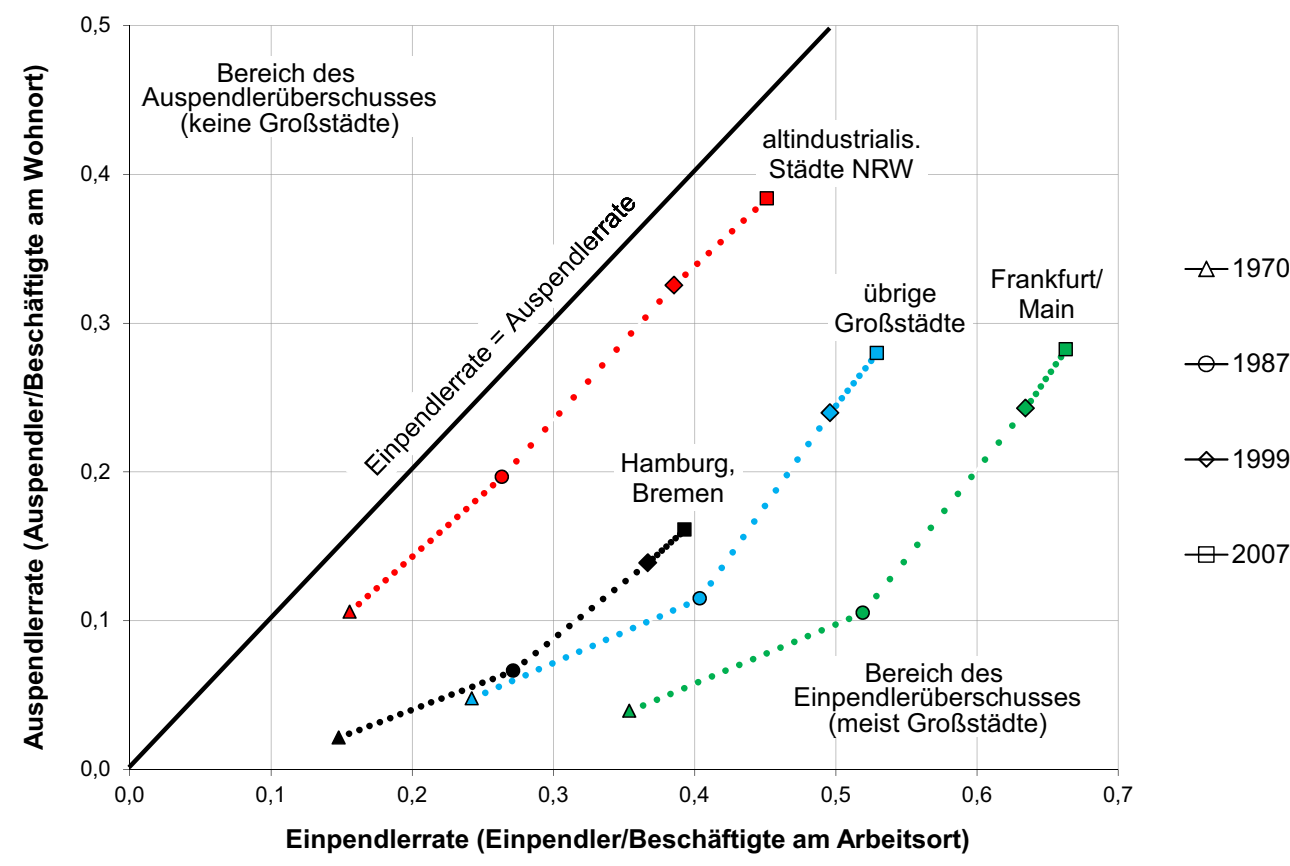

übrige Großstädte

Bielefeld, Bonn, Düsseldorf, Hannover, Köln, Mannheim, München, Nürnberg, Stuttgart

\begin{abstract}
Der Abstand der Punktierung entspricht der mittleren jährlichen Veränderung zwischen den Jahren 1970, 1987, 1999 und 2007.

geringer Abstand = geringe Zunahme/Jahr großer Abstand = große Zunahme/Jahr
\end{abstract}

landgemeinden (Kagermeier 1997: 21 ff.; Motzkus 2002: 112 ff.; Siedentop/Stein/Lanzendorf 2005: 95 ff.). Folgende Interpretation liegt nahe: In den kleineren Gemeinden fehlt vieles von dem, was einen verkehrssparsamen Alltag möglich macht. Die planerische Schlussfolgerung lautet: Wenn sich die Randwanderung auf die größeren Umlandgemeinden richten würde, würde dies Verkehr sparen (vgl. die Diskussion des Konzepts „dezentrale Konzentration“ bei Siedentop/Stein/Lanzendorf 2005: 42 ff.). Nach Aktionen differenziert sind diese Unterschiede vor allem auf den Berufsverkehr zurückzuführen (Geier/Holz-Rau/Krafft-Neuhäuser 2001). Eine Unterscheidung von Alteingesessenen und Neubürgern zeigt aber, dass die Unterschiede gerade im Berufsverkehr zwischen den Gemeindetypen unter den Zugezogenen relativ gering sind. Wer aus der Kernstadt ins Umland zieht, arbeitet typischerweise weiterhin in der Kernstadt (Geier/Holz-Rau/Krafft-Neuhäuser 2001; Bauer/ Holz-Rau/Scheiner 2005; Scheiner 2009: 162 ff.). Da aber der Anteil der Zugezogenen in den kleineren Gemeinden wesentlich höher als in den größeren Umlandgemeinden ist, legt die Bevölkerung der kleineren Umlandgemeinden weitere Wege zurück als die Bevölkerung der größeren Umlandgemeinden. Wer also die Randwanderung erfolgreich in die größeren Umlandgemeinden lenkt, erreicht in der Gesamtbetrachtung des Umlandes zumindest im für die
Distanzunterschiede besonders relevanten Berufsverkehr keinen wesentlichen Verkehrsspareffekt, möglicherweise aber einen Verlagerungseffekt zum ÖPNV (Einschränkungen; vgl. das Gedankenexperiment oben). Unbenommen sind diese Standorte im Einkaufs- und Schülerverkehr verkehrssparsamer. Für die Gesamtbilanz der Alltagsdistanzen ist dies aber kaum relevant. ${ }^{3}$

Bei Analysen von Zeitschnitten geraten Veränderungsprozesse leicht aus dem Blickfeld. Dies verdeutlichen die bundesweiten Berufspendlerstatistiken von 1970 bis 2007. In diesem Zeitraum sind die Pendlerverflechtungen der deutschen Großstädte ${ }^{4}$ deutlich gestiegen, am stärksten im Zeitraum zwischen 1987 und 1999 (vgl. Abb. 2). Dies betrifft die Einpendlerraten und die Auspendlerraten, letztere in der jüngeren Vergangenheit sogar in besonderem Maße. Neben der Randwanderung des Wohnens und der Arbeitsplätze nimmt die bindende Kraft von Arbeitsplätzen über die Zeit deutlich ab. Der Kern des Prozesses ist dabei eher durch die steigende Motorisierung, durch eine

\footnotetext{
${ }_{3}$ Dies stellt nicht infrage, dass entsprechende Angebote zur Erreichbarkeitssicherung für Personen ohne Auto oder ohne Autoaffinität im Alltag beitragen und daher sinnvoll sind.

${ }^{4}$ Wegen der längeren Zeitreihe werden hier nur die Städte der alten Bundesländer betrachtet.
} 
Tab. 1 Durchschnittsdistanzen des Alltagsverkehrs im Zeitverlauf (km pro Person und Jahr, jeweilige Minima in Kursiv)

\begin{tabular}{|c|c|c|c|c|c|}
\hline Gemeindegrößenklasse $^{a}$ & 1976 & 1982 & $1989^{\mathrm{b}}$ & 2002 & 2008 \\
\hline Unter 5.000 Einwohner & 10.150 & 11.876 & 12.937 & 13.539 & 15.485 \\
\hline 5.000 bis unter 20.000 Einwohner & 9.730 & 11.414 & 12.260 & 13.246 & 14.120 \\
\hline 20.000 bis unter 50.000 Einwohner & 8.614 & 11.394 & 11.304 & 11.637 & 13.424 \\
\hline 50.000 bis unter 100.000 Einwohner & & 9.752 & 11.293 & 11.294 & 12.883 \\
\hline 100.000 bis unter 500.000 Einwohner & 8.978 & 10.597 & 10.872 & 11.969 & 12.430 \\
\hline 500.000 bis unter 1 Mio. Einwohner & 9.270 & 10.522 & 10.740 & 10.087 & 12.754 \\
\hline ab 1 Mio. Einwohner & & 11.690 & & 11.591 & 10.961 \\
\hline Insgesamt & 9.329 & 11.066 & 11.520 & 12.363 & 13.558 \\
\hline
\end{tabular}

${ }^{a}$ Die Kategorisierung der Gemeindegröße erfolgte nicht in allen Erhebungen identisch. Daher kommt es hier in einzelnen Jahren zu unterschiedlichen Kategoriengrenzen

${ }^{b}$ Die Originalwerte der Erhebung aus dem Jahr 1989 weichen methodenbedingt von den anderen Werten der Zeitreihe nach unten ab. Die Distanzschätzung wurde daher um den Faktor 1,21 erhöht. Dieser Wert passt den Gesamtdurchschnitt plausibel in die Zeitreihe ein. Dies hat keine Auswirkungen auf den Vergleich der Mittelwerte nach Gemeindegrößenklassen

Quelle: eigene Analysen der KONTIV 1976, 1982 und 1989 sowie der Studien „Mobilität in Deutschland“ 2002 und 2008

generell zunehmende Raumdurchlässigkeit und vor allem durch Veränderungen auf dem Arbeitsmarkt (Spezialisierung, Zeitverträge) geprägt als durch die Verteilung von Arbeitsplätzen und Wohnstandorten im Raum, denn die Pendelnotwendigkeit auf der Basis der regionalen Verteilung von Wohnen und Arbeiten hat kaum zugenommen (Guth/Siedentop/Holz-Rau 2012: 494). Dies lässt sich auch als Prozess einer Entkopplung des Verkehrsverhaltens von der gebauten Umwelt des Nahbereichs beschreiben (HolzRau 1997; Schmitz 2001).

Auch die oben dargestellten Unterschiede des Verkehrsverhaltens nach der Gemeindegröße sind zeitlich nicht stabil. So zeigten sich 2008 die geringsten Tagesdistanzen in den Millionenstädten und die höchsten in den Gemeinden unter 5000 Einwohnern (vgl. Tab. 1). In den Erhebungswellen vorher (nur alte Bundesländer) lag das Minimum dagegen in kleineren Großstädten, während die Bevölkerung der Millionenstädte, aber auch der kleineren Gemeinden weitere Wege zurücklegte (vgl. Tab. 1). Seit der ersten KONTIV-Erhebung ${ }^{5} 1976$ haben die Durchschnittsdistanzen in den kleineren Gemeinden am stärksten, in den Millionenstädten kaum oder gar nicht zugenommen. So hat sich seit 1976 das Minimum allmählich von kleineren Großstädten hin zu den Millionenstädten verschoben bei einem gleichzeitig deutlichen Anstieg der Durchschnittsdistanzen in allen Gemeindegrößenklassen. Eine Extrapolation in die Vergangenheit lässt vermuten, dass in den 1950er- und 1960er-Jahren die Durchschnittsdistanzen in den Millionenstädten sogar am höchsten und in den kleinsten Gemeinden am geringsten waren. In der DDR legte die Bevölkerung der Umlandgemeinden noch in den 1980er-Jahren kürzere Distanzen zurück als die Bevölkerung der Kernstädte (Gertz/ Holz-Rau/Rau 1994: 38). In vielen Großstädten der alten Bundesländer war die Motorisierung ausgesprochen hoch,

\footnotetext{
5 Kontinuierliche Erhebung zum Verkehrsverhalten.
}

im ländlichen Raum eher gering (Scheiner 2012: 319). Ein weiterer wesentlicher Treiber neben der zunehmenden Motorisierung waren Veränderungen der Wirtschaftsstruktur. So waren viele Erwerbstätige in den kleineren Gemeinden um 1960 noch in der Landwirtschaft beschäftigt. Die Berufswege waren kurz und führten meist nicht aus der eigenen Gemeinde hinaus. Erst der Bedeutungsverlust der Landwirtschaft führte zu einer rapiden Zunahme der Pendelströme in die Großstädte. Außerdem nimmt der Grad der Spezialisierung auf dem Arbeitsmarkt zu und es wird auch in größeren Gemeinden immer schwieriger, einen passenden Arbeitsplatz zu finden. Dies wird noch verstärkt durch die zunehmende Doppelerwerbstätigkeit in Haushalten.

Bezogen auf den Regelkreis nach Wegener (2009) bedeutet dies, dass ein „Zurückdrehen des Regelkreises“ nicht zu den ursprünglichen Verkehrsstrukturen führt. Wir würden heute die Siedlungsstrukturen der Vergangenheit deutlich verkehrsaufwendiger nutzen als damals.

\subsection{Zur Gestaltbarkeit der Ursachen}

Um die Verkehrsentwicklung erfolgreich zu beeinflussen, genügt es nicht, (raumstrukturelle) Ursachen zu identifizieren. Vielmehr müssen diese Ursachen auch zielgerichtet gestaltbar sein. Dabei bietet aber nicht jede Kausalität auch den Ansatz zur Steuerung.

Die deutlich geringeren Alltagsdistanzen der Bevölkerung der (Innen-)Städte resultieren aus deren zentralörtlichem Bedeutungsüberschuss. Eine Region kann aber nicht nur aus Innenstädten bestehen. Wer also aufgrund der beobachteten Unterschiede im Verkehrsverhalten zwischen Innenstadt und Stadtrand oder Umland auf ein Verkehrssparpotenzial schließt, verkennt die Bedeutung der Arbeitsteilung innerhalb von Regionen, die sich in diesem Befund niederschlägt. Selbst existierende kausale Zusammenhänge 
lassen sich also nicht unbedingt zur Verkehrsvermeidung instrumentalisieren.

Die Planungskonzepte unterschiedlicher Sektoren stehen häufig in Konkurrenz zueinander. Trotz der aus heutiger Sicht erheblichen Verkehrsnachteile der Randwanderung von Wohnstandorten entlastete diese Entwicklung die städtischen Wohnungsmärkte. Die Randwanderung von Betrieben, vor allem im produzierenden Sektor, verbesserte deren Wirtschaftlichkeit und gleichzeitig die innerstädtische Wohnqualität. So entstanden in den (Innen-)Städten und Regionen Potenziale für den steigenden Wohnflächenbedarf und Flächenpotenziale für tertiäre und quartäre Arbeitsplätze an teils hervorragend ÖPNV-erschlossenen Standorten. Eine Umkehr dieser Entwicklung ließe sich bei den heutigen Flächenansprüchen für das Wohnen (vgl. zu den Trägergruppen der Reurbanisierung Kabisch/Steinführer/Haase 2012, speziell zu Familien Frank 2013, zur Situation in den „Hauptstädten“ der Reurbanisierung Herfert/Osterhage 2012) sowie für die Produktion und Distribution im Rahmen einer verträglichen Dichte in den meisten Städten wohl nicht realisieren und wäre allein anhand des Verkehrsvermeidungspotenzials auch nicht zu rechtfertigen.

Auch Konzepte, die an der Raumdurchlässigkeit ansetzen, ein kapazitätsreduzierender Rückbau des Hauptverkehrsstraßennetzes, nicht nur einzelner Straßenabschnitte (der unseres Wissens nirgends diskutiert wird) sowie eine drastische Verteuerung des Autoverkehrs erscheinen politisch kaum mehrheitsfähig. Darüber hinaus gibt es eine Vielzahl gesellschaftlicher Prozesse mit Auswirkungen im Verkehrsbereich. Der Bedeutungsverlust der Landwirtschaft und Spezialisierungen auf dem Arbeitsmarkt wurden oben bereits angesprochen. Diese Prozesse unterliegen einer starken Eigendynamik mit sehr begrenzten politischen Steuerungsmöglichkeiten (Rammler 2001). Wo entsprechende Handlungsmöglichkeiten bestehen, sind diese Prozesse trotz ihrer verkehrsgenerierenden Wirkung politisch eher gewünscht als unerwünscht und werden entsprechend politisch unterstützt. Dies betrifft insbesondere Entscheidungsprozesse auf der Ebene des Bundes und der EU, die sich nicht an den Vorstellungen einer integrierten Planung, sondern überwiegend am Ziel des Wirtschaftswachstums orientieren und hierfür den großräumigen Austausch von Waren (Güterverkehr) und Arbeitskräften (Personenverkehr) gezielt fördern.

Die Zunahme der Distanzen als dominanter Entwicklung im Verkehrsbereich (mindestens) der letzten Jahrzehnte ist danach nur eingeschränkt aus den Veränderungen der gebauten Umwelt zu erklären (auch wenn er mit diesen verbunden ist). Die Ausweitung der Aktionsräume ist gleichzeitig und unserer Ansicht nach dominant mit anderen Aspekten des gesellschaftlichen, vor allem des sozialen Wandels verbunden (vgl. Kap. 5). Diese Prozesse liegen aber überwiegend außerhalb des Handlungsfeldes der Stadt- und Regionalentwicklung und werden gemeinhin als ,gesellschaftlicher Fortschritt“ begrüßt. Entsprechend erscheint ein Rückdrehen dieser Entwicklung ebenfalls kaum ein geeigneter Ansatz zur Verkehrsvermeidung.

\section{Sozialer Wandel und Verkehr}

Der soziale Wandel der letzten Jahrzehnte wurde mit einer Vielzahl von Kernbegriffen wie Modernisierung und Individualisierung umfassend beschrieben (für den Verkehrskontext vgl. Rammler 2001; Canzler/Kaufmann/Kesselring 2008; Scheiner 2009). Seine wesentlichen Teilprozesse wirken im Prinzip alle in eine Richtung: in Richtung eines zunehmenden Verkehrsaufwandes, einer Ausdehnung der Aktionsräume im privaten Personenverkehr und der Verflechtungsräume im Güter- und Wirtschaftsverkehr (Axhausen 2007). Für den Personenverkehr sei dies hier beispielhaft anhand von vier Entwicklungen beschrieben. Dabei werden teilweise die in den Analysen des Verkehrsverhaltens bisher meist ausgeblendeten Fernreisen einbezogen. Dies geschieht aus zwei Gründen:

Erstens trägt der Fernverkehr mit mehr als $50 \%$ erheblich zu den klimarelevanten Emissionen des Personenverkehrs bei (vgl. für Deutschland Aamaas/Borken-Kleefeld/ Peters 2013; für Finnland am Beispiel von Helsinki Ottelin/Heinonen/Junnila 2014). Überlegungen zum Beitrag des Verkehrssektors zur Reduzierung klimarelevanter Emissionen sollten daher diesen besonderen Wachstumsbereich des Verkehrs umfassen. Auch wenn Interventionen in die gebaute Umwelt nicht mit möglichen Wirkungen auf den Fernverkehr begründet werden, ist dieser zur Einordnung des möglichen Wirkungsumfangs relevant. Dies gilt umso mehr, da mit der Zunahme der Fernverkehre, insbesondere des Fernpendelns (Pütz 2015: 14 f.), ein immer größerer Teil der zurückgelegten Distanzen und damit der klimarelevanten Verkehrsemissionen aus vielen städtischen und regionalen Verkehrsanalysen ausgeblendet werden.

Zweitens sind die im Alltag verkehrssparsamen Städter besonders fernreiseaktiv. Dies ist im Sinne einer Selbstselektion von Bevölkerung und Wirtschaft ein Hinweis auf sozialräumliche Konfigurationen, die nur in der Analyse des Gesamtverkehrs richtig zu interpretieren sind. Dies gilt auch für die aktuelle, teils ökologisch erfreute Debatte um die abnehmende Pkw-Nutzung junger Erwachsener. Diese könnte zu ganz anderen Schlussfolgerungen führen, wenn man gleichzeitig feststellt, dass diese besonders fernreiseaktiv sind (vgl. für Hinweise in diese Richtung Frändberg 2009).

Die folgenden Ergebnisse stützen sich aufgrund der Datenlage überwiegend auf Erhebungen im zeitlichen Querschnitt. Dabei besteht das grundsätzliche Problem der Fehlinterpretationen von Querschnittsanalysen (vgl. 
Kap. 3). Allerdings zeigen sich ähnliche Befunde in zahlreichen Untersuchungen zu unterschiedlichen Zeitpunkten in verschiedenen Ländern (Holz-Rau 1997; Schwanen/Dijst/ Dieleman 2004; Scheiner 2009; Ewing/Cervero 2010; Næss 2011; Elldér 2014; van Wee/Handy 2016) sowie innerhalb Deutschlands zwischen den unterschiedlichen Wellen der KONTIV/Mobilität in Deutschland (MiD)-Erhebungen (Konrad 2016). Außerdem sind uns keine theoretischen Zweifel an diesen Zusammenhängen bekannt. In Kap. 3 konnte dagegen bezogen auf die Zusammenhänge zwischen der gebauten Umwelt und dem Verkehrsverhalten gezeigt und begründet werden, dass und warum diese Zusammenhänge zeitlich nicht stabil sind.

\subsection{Höhere Einkommen = höhere Motorisierung und Distanzen}

Sowohl im Alltags- als auch im Fernverkehr zeigen sich deutliche Zusammenhänge zwischen Haushaltseinkommen und Reisedistanzen sowie Verkehrsmittelnutzung. So beträgt die Differenz nach einer multivariaten Analyse zwischen der höchsten und niedrigsten Einkommenskategorie (oberste und unterste 10\%) im Alltagsverkehr $3.500 \mathrm{~km}$ pro Person und Jahr und im Fernverkehr $7.700 \mathrm{~km}$ pro Person und Jahr (Holz-Rau/Scheiner/Sicks 2014: 498) bei einem Durchschnitt von 12.500 bzw. 7.700 km pro Person und Jahr (Holz-Rau/Scheiner/Sicks 2014: 500). Diese Unterschiede betreffen insbesondere die Reisen mit dem Pkw und Flugzeug (Reichert/Holz-Rau 2014). Entsprechend ist der längerfristige Wohlstandszuwachs mit einer Zunahme der Distanzen verbunden (Dargay 2007), im Alltagsverkehr vor allem mit dem motorisierten Individualverkehr, im Fernverkehr vor allem mit dem Flugzeug.

Bei genauerer Betrachtung sind mindestens drei Aspekte zu unterscheiden:

- Die allgemeine Zunahme der Einkommen bzw. Kaufkraft eröffnet finanzielle Spielräume für die private Motorisierung und Reisen. Sie bildet eine Voraussetzung für die Ausdehnung der Aktionsräume im Alltag sowie für Urlaubsreisen.

- Die Zunahme der Einkommen resultiert neben allgemeinen Gehaltssteigerungen auch aus einer Zunahme höher qualifizierter Tätigkeiten. Letzteres ist verbunden mit einer höheren Häufigkeit von Dienst- und Geschäftsreisen.

- Die gleichzeitige Reduzierung der Kosten im Flugverkehr ermöglicht die Zunahme der Dienstreisen und Privatreisen mit dem Flugzeug und damit auch über längere Strecken.

Höhere Einkommen sind mit höheren Distanzen im Alltags- und im Fernverkehr verbunden. Eine Zunahme der Einkommen dürfte daher zur Zunahme der Reisedistanzen im Alltags- und Fernverkehr beigetragen haben und weiter beitragen.

\subsection{Höhere Bildung und höhere Spezialisierung = höhere Distanzen}

Zu den oben dargestellten Einkommensunterschieden nahezu parallele empirische Befunde zeigen sich (unter gleichzeitiger Berücksichtigung der Einkommenseffekte) nach Bildungsniveau (nur Personen nach Abschluss ihrer Ausbildungsphase). So legen Personen mit Hochschulabschluss jährlich im Alltagsverkehr $1.700 \mathrm{~km}$ und im Fernverkehr $7.200 \mathrm{~km}$ mehr zurück als Personen mit Hauptschulabschluss oder ohne Schulabschluss (Holz-Rau/Scheiner/ Sicks 2014: 498). Höhere Bildungsabschlüsse bedeuten eine höhere Spezialisierung auf dem Arbeitsmarkt, damit eine geringere Dichte passender Angebote und in der Folge häufig längere Berufswege.

Neben den besseren Sprachkenntnissen bei höherer Bildung, die gleichzeitig Kompetenz und Anreiz für Auslandsreisen sein können, werden Auslandsaufenthalte selbst zum Bestandteil der Ausbildungsphase (HIS/DAAD 2013). Vieles spricht dafür, dass diese Auslandsaufenthalte später private Besuche und einen höheren Anteil internationaler Berufsmobilität nach sich ziehen, die insgesamt zu einer privaten Globalisierung führen (Frändberg/Vilhelmson 2003; Frei/Axhausen/Ohnmacht 2009). Dies trägt zu einer erheblichen Ausweitung der Fernreisen bei.

Höhere Bildung (und die parallele Spezialisierung vor allem des Arbeitsmarktes) sind mit höheren Distanzen im Alltags- und Fernverkehr verbunden. Auch die Bildungsexpansion dürfte damit zu einer Erhöhung der Distanzen im Alltags- und Fernverkehr beigetragen haben und weiter beitragen.

\subsection{Gleichstellung der Frau = höhere Motorisierung und Distanzen}

Die Zunahme der privaten Motorisierung der letzten Jahrzehnte ist vor allem auf die Zunahme der Motorisierung von Frauen zurückzuführen. In den jüngeren Kohorten finden sich bei gleicher Erwerbstätigkeit kaum noch Unterschiede bei Pkw-Verfügbarkeit, Distanzen und Pkw-Nutzung zwischen Frauen und Männern (Konrad 2016). Gleichzeitig hat die Erwerbstätigkeit der Frauen deutlich zugenommen. Erwerbstätigkeit ist bei Männern wie Frauen mit höheren Distanzen und stärkerer Pkw-Nutzung verbunden (Konrad 2016). (Noch) bestehende Unterschiede im Fernverkehr sind auf die höhere Geschäftsreisetätigkeit von Männern zurückzuführen, die auf unterschiedliche Berufsprofile von Männern und Frauen hinweisen (Holz-Rau/Scheiner/Sicks 2014: 497 ff.). 
Darüber hinaus erschwert die Doppelerwerbstätigkeit in Haushalten eine Pendeldistanzen minimierende Wohnstandortwahl. Unabhängig davon, wie die Pendelbelastung in Paaren aufgeteilt wird - meist sind die Wege der Männer länger als die Wege der Frauen - ist es für Haushalte mit zwei Erwerbstätigen grundsätzlich schwieriger, Wohn- und Arbeitsorte verkehrssparsam aufeinander abzustimmen als für Haushalte mit nur einem Erwerbstätigen. Empirische Studien zeigen hierzu allerdings inkonsistente Ergebnisse (Sultana 2005; Surprenant-Legault/Patterson/El-Geneidy 2013).

Eine Angleichung wesentlicher Verkehrskenngrößen hat sich in den jüngeren Kohorten weitgehend auf dem ursprünglich höheren Niveau der Männer vollzogen (Beckmann/Holz-Rau/Rindsfüser et al. 2005; Frändberg/Vilhelmson 2011; Konrad 2016). Erst im letzten Jahrzehnt gibt es in einigen Ländern Anzeichen für eine Abnahme der PkwNutzung und einen Rückgang der Alltagsdistanzen unter jungen Männern (Frändberg/Vilhelmson 2011; Kuhnimhof/ Armoogum/Buehler et al. 2012). Ob die Abnahme der Distanzen auch unter Einbeziehung von Fernreisen gilt, ist bisher nicht untersucht und erscheint uns unwahrscheinlich.

Insgesamt ist der Wandel des Geschlechterverhältnisses mit einer zunehmenden Berufstätigkeit und Motorisierung der Frauen verbunden. Beides führt zu einer Zunahme der Distanzen im Alltag.

\subsection{Virtualisierung, Multilokalisierung und statushomogene Partnerschaften $=$ höhere Distanzen}

Private (und berufliche) Netzwerke lassen sich mit Telekommunikationsmitteln heute leichter als noch vor wenigen Jahren auch über große Entfernungen bilden und aufrechterhalten, z. B. bei jungen Erwachsenen die Kontakte aus einem Auslandsstudium. Dies ist auch eine Bedingung für die Zunahme einer Vielzahl von Formen multilokalen Wohnens, die mit entsprechenden räumlichen Austauschbeziehungen und langen Wegen verbunden sind (Living Apart Together-Paare, pendelnde Kinder in Nachtrennungsfamilien, Fernpendler-Ehen etc.; vgl. Hesse/Scheiner 2007). Die Bildung von Partnerschaften unter den Bedingungen großer Raumdurchlässigkeit und großer, auch virtueller Suchräume - über Internet-Partnerbörsen - fördert die Statushomogenität von Paaren. Gleichzeitig wird damit die soziale Polarisierung auf der Haushaltsebene wesentlich verstärkt (Blossfeld/Timm 2003).

Die Ausdehnung der Suchräume für persönliche Beziehungen führt zu höheren zurückgelegten Distanzen, vor allem zum Fernpendeln und Wochenendreisen.

\section{Zusammenfassung und Ausblick}

Verkehrs- und Raumentwicklung bilden einen sich gegenseitig bedingenden Prozess, der im Planungsdiskurs häufig als Regelkreis beschrieben wird. Der Regelkreis von Wegener (2009) und ähnliche Modellvorstellungen betonen die wechselseitigen Zusammenhänge der Standort- und Verkehrsentscheidungen sowie der Standort- und Verkehrsentwicklung und damit die Bedeutung der Standort- und Verkehrsplanung. Dies gilt auch für die (meist vierstufigen) Verkehrsberechnungsmodelle, in denen das unterschiedliche Verkehrsverhalten in spezifischen Konfigurationen der gebauten Umwelt zur Grundlage von Verkehrsprognosen und Wirkungsschätzungen gemacht wird. Bezogen auf den aktuellen Kenntnisstand lässt sich dazu festhalten: Wesentliche Bedingungen für die vermuteten Potenziale der Verkehrsvermeidung einer integrierten Standort- und Verkehrsplanung, insbesondere der Planung der gebauten Umwelt, und ihre Quantifizierung in Verkehrsmodellen sind nicht erfüllt. Es fehlt an methodisch robusten Belegen für die Kausalität von Zusammenhängen und an Wissen über die zeitliche Stabilität der beobachteten Beziehungen. Gleichzeitig sind die Möglichkeiten zur planerischen Gestaltung der vermuteten Ursachebereiche gering.

Beobachtete Unterschiede im Verkehrsverhalten zwischen unterschiedlichen räumlichen Kategorien beruhen teilweise auf räumlichen Unterschieden der Bevölkerungsstruktur, stehen im Zusammenhang mit Prozessen der Selbstselektion und lösen die aufeinander bezogenen räumlichen Einheiten auf, obwohl diese nur als Gesamtraum funktionsfähig sind.

Die räumlichen Unterschiede des Verkehrsverhaltens sind zeitlich nicht stabil und werden entscheidend durch den gesellschaftlichen Wandel geprägt. Dabei verleiten Analysen im zeitlichen Querschnitt dazu, räumliche Unterschiede im Verkehrsverhalten aus ihrem Entstehungsprozess zu lösen und falsch zu interpretieren. Gegenüber den räumlichen Unterschieden zu einem gegebenen Zeitpunkt dominiert aber das Verkehrswachstum im Zeitverlauf.

Die beschriebenen Prozesse gesellschaftlicher Veränderungen führen alle zu einem höheren Verkehrsaufwand. Dies gilt aktuell insbesondere für den in der Verkehrsforschung häufig ausgeblendeten Fernverkehr (vgl. als Ausnahmen etwa Holden/Linnerud 2011; Holz-Rau/Scheiner/ Sicks 2014) sowie den auch hier nicht betrachteten Güterverkehr.

Wesentliche Aspekte, die wir gemeinhin als gesellschaftlichen Fortschritt betrachten - Wirtschaftswachstum und Wohlstand, Wandel des Geschlechterverhältnisses und Bildungsexpansion, Globalisierung und Reisen - führen zu einer Ausweitung der regionalen, überregionalen und weltweiten Interaktionen. Diese Trends werden gesellschaftlich weitgehend - und teilweise sogar seitens einer ökologisch 
orientierten Wissenschaft ${ }^{6}$ - begrüßt und durch die Politik gefördert.

Auch die geringere (aber weiterhin hohe) Pkw-Nutzung jüngerer Erwachsener und die Bevölkerungszunahme in den Großstädten, die in der Mobilitätsforschung teilweise als Anzeichen einer Verkehrswende betrachtet werden (peak car, peak travel) (vgl. Millard-Ball/Schipper 2011; Kuhnimhof/Armoogum/Buehler et al. 2012; van Wee 2015), lassen auch pessimistischere Interpretationen zu:

Junge Erwachsene könnten besonders fernreiseaktiv sein und so, trotz geringerer Pkw-Nutzung, überdurchschnittlich zu den verkehrsbedingten klimarelevanten Emissionen beitragen. Für diejenigen, die besonders fernreiseaktiv sind, mehrere Wohnsitze, wechselnde Arbeitsorte, großräumige soziale und berufliche Netze haben, sind flexiblere Angebote wie beispielsweise das Carsharing eine alltagstauglichere Variante als der eigene Pkw. Die geringere Pkw-Nutzung junger Erwachsener kann also auch auf eine weitere Ausdehnung der Aktionsräume über die Einsatzbereiche des Pkw hinaus hinweisen - im Sinne des Satzes eines unserer ehemaligen Studenten: „Wir haben kein eigenes Auto, weil man mit einem Auto nicht fliegen kann“ (Björn Vetter). Zukünftige Untersuchungen in diesem Kontext dürfen sich daher nicht auf den Alltagsverkehr beschränken, sondern sollten Fernreisen sowie Entwicklungen wie die zunehmende Multilokalität beim Wohnen und Arbeiten einbeziehen.

Das Wachstum der Kernstädte ist in vielen Regionen mit dem gleichzeitigen Wachstum der besonders alltagsverkehrsaufwendigen Umlandgemeinden verbunden. Eine Reurbanisierung zulasten des Umlandes findet am ehesten in den neuen Bundesländern statt (Herfert/Osterhage 2012) und betrifft damit in Deutschland nur wenige Räume. Die Fokussierung auf das Thema Reurbanisierung blendet dieses Wachstum in den besonders alltagsverkehrsaufwendigen Randbereichen aus. Dabei kann der Bedeutungsgewinn der Verdichtungsräume insgesamt trotz zunehmender Bevölkerungszahlen in den Kernstädten mit einer weiteren Zunahme des Verkehrsaufwandes verbunden sein.

Aus Verkehrssicht stellt sich ergänzend die Frage, ob das Verkehrsverhalten der „Reurbaniten“ dem Verkehrsverhalten entspricht, das bisher für die Bevölkerung der (Innen-) Städte charakteristisch war. Ist der Drang in die (Innen-) Städte unter Umständen sogar Ausdruck der mit einer Doppelerwerbstätigkeit hochqualifizierter Paare verbundenen Multilokalität, des zeitaufwendigen Pendelns zwischen Orten hoher Zentralität (Pütz 2015: 8 f.), einer höheren Bedeutung von (privaten wie geschäftlichen) Fernreisen?

\footnotetext{
${ }^{6}$ So etwa wenn van Wee und Handy (2016: 21) den positiven Nutzen des induzierten Verkehrs betonen, der durch sinkende Verkehrskosten entsteht und konsequenterweise fordern, diesen in Bewertungsverfahren einfließen zu lassen. Faktisch passiert ja genau dies in den KostenNutzen-Analysen der Bundesverkehrswegeplanung.
}

Die stark wachsenden Zentren sind jedenfalls meist besonders gut in die Fernverkehrsnetze eingebunden und die besonders fernreiseaktive Bevölkerung konzentriert sich in diesen Agglomerationsräumen.

Wir vermuten, dass der Trend zu einer verkehrsaufwendigeren Gesellschaft ungebrochen ist, sich aber zunehmend jenseits des Einsatzbereichs des Pkw realisiert. Die Räume, in denen sich diese Entwicklung besonders ausprägt, sind die hochverdichteten Räume, deren Zentren wie auch deren Peripherie. Die aktuellen Prozesse der Reurbanisierung oder, unseres Erachtens zutreffender, des Wachstums der Agglomerationen insgesamt, und die geringere PkwNutzung junger Erwachsener sind mit einem weiter steigenden Verkehrsaufwand verbunden und nicht die ersten Anzeichen einer verkehrssparsameren Verkehrswende. So mag der Pkw-Verkehr in diesem Prozess etwas abnehmen - der Verkehrsaufwand insgesamt und damit auch die klimarelevanten Emissionen werden durch die Zunahme besonders langer Distanzen aber weiter steigen. Bezieht man sich hier noch einmal auf den eingangs dargestellten Regelkreis von Wegener (2009), so handelt es sich um eine Spirale und nicht um einen in sich geschlossenen Kreis. In dieser Spirale führen viele gesellschaftliche Entwicklungslinien zu einem höheren Verkehrsaufwand, viel mehr als nur die Raumentwicklung.

Die Überprüfung dieser Hypothese zur Dynamik von Gesellschaft, Raum und Verkehr erfordert erweiterte theoretische und empirische Konzepte der sozialen und räumlichen Differenzierung, etwa unter dem Begriff der Netzwerkgesellschaft (vgl. Castells 2004), die Einbeziehung von Präferenzen und Lebensstilen, von Multilokalitäten, eine verbesserte Raumbeobachtung, Geocodes in Verkehrsdaten und Paneldaten. Dabei kann es gleichzeitig sinnvoll sein, die Analysen sozial feiner zu differenzieren, aber auch räumlich zu aggregieren:

- Gibt es Personen(gruppen), die sich tatsächlich vom bisherigen Pfad steigenden Verkehrsaufwandes abkoppeln?

- Finden sich neben verkehrssparsamen und verkehrsaufwendigen Teilräumen auch ganze Regionen, die insgesamt verkehrssparsam oder verkehrsaufwendig organisiert sind?

Wenn man dieser Einschätzung folgt, führt dies zu einer Verschiebung von Prioritäten in der Diskussion über den Beitrag einer integrierten Standort- und Verkehrsplanung zum Klimaschutz, die hier nur angerissen werden können. Dabei zweifeln wir nicht daran, dass eine zukunftsfähige Entwicklung von Städten und Regionen eine integrierte Standort- und Verkehrsplanung, orientiert an einem Leitbild nutzungsgemischter und kompakter Strukturen, benötigt. Aber es ergibt keinen Sinn, dies falsch zu begründen und unrealistische Ziele anzustreben. Verkehrsplanung ist heute gekennzeichnet durch extrem breit definierte, unklare 
und nebulöse Ziele (Taylor/Morris 2015). Dies steht einer erfolgreichen Planung in den Regionen und Kommunen im Weg und kann sogar dem Klimaschutz schaden, weil die Misserfolge erst nach sehr langer Zeit deutlich zutage treten. Die Reduktion von $\mathrm{CO}_{2}$-Emissionen ist nur eines unter vielen Zielen einer integrierten Standort- und Verkehrsplanung. Die vorrangigen Interventionsmöglichkeiten liegen auf der Ebene des Bundes und der EU. Im Mittelpunkt einer integrierten Standort- und Verkehrsplanung stehen dagegen eine hohe Lebensqualität in Städten und Regionen, Teilhabe- und Teilnahmechancen für alle, hohe Verkehrssicherheit (als Perspektive keine Verkehrstoten), gesunde Umgebung (ohne Belastungen der Gesundheit durch Verkehrslärm und andere verkehrsbedingte Immissionen), öffentliche Räume mit kommunikativer und kultureller Anregung sowie geringe Kosten für die (auch zukünftige) Allgemeinheit sowie für sozial benachteiligte Nutzergruppen.

Zur Erreichung dieser Ziele wären deutlich stärker als bisher nicht nur Angebotsverbesserungen für planerisch erwünschte Verkehrsmittel (Pull-Strategien), sondern Einschränkungen für unerwünschte Verkehrsmittel (PushStrategien) erforderlich, z.B. Parkraumeinschränkungen (Petrunoff/Rissel/Wen et al. 2015). Diese sind deutlich konfliktreicher als bloße Angebotsverbesserungen (eventuell sogar parallel für alle Verkehrsmittel).

Entsprechend lauten die Strategien weiterhin, allerdings orientiert an realistischen Zielen und adressiert an die jeweils relevanten Akteure und Ebenen:

- Erreichbarkeit ohne Pkw sichern durch eine integrierte Standort- und Verkehrsplanung mit barrierefreien Verkehrsangeboten und Gelegenheiten, mit Handlungsoptionen im Nahraum, mit Standortoptionen ohne strukturelle Pkw-Abhängigkeit auf der Ebene von Kommunen und Regionen,

- Verkehr verträglicher abwickeln (sicherer, leiser, weniger Emissionen) als Schadensbegrenzung und Qualitätsverbesserung durch vor allem ordnungspolitische Rahmensetzungen auf der Ebene des Bundes und der EU (besonderes Augenmerk auf den Flugverkehr), durch $\mathrm{CO}_{2-}$ Kompensationszahlungen oder erheblich erhöhte Kraftstoffkosten sowie bezogen auf die Belastungen vor Ort durch verkehrsplanerische Interventionen auf der Ebene von Kommunen und Regionen,

- Verkehr verlagern als lokale und regionale Option entsprechend der Zielsetzungen der kommunalen und regionalen Standort- und Verkehrsplanung (z. B. Urbanität und Stadtraumqualität durch weniger Kfz-Verkehr, mehr Rad- und Fußverkehr) und gleichzeitig als Nebeneffekt von Maßnahmen zur besseren Erreichbarkeit mit einem allerdings nur geringen Beitrag zum Klimaschutz,

- Verkehr vermeiden als langfristige Hoffnung auf veränderte Einstellungen zum Verkehr (wohl nur zum Alltags- verkehr) und gleichzeitig als Nebeneffekt von Maßnahmen zur besseren Erreichbarkeit mit einem nur geringen Beitrag zum Klimaschutz auf kommunaler und regionaler Ebene. Ergänzend könnten hierzu auch erheblich erhöhte Kraftstoffkosten beitragen, wenn diese umsetzbar wären (wir halten dies für unwahrscheinlich). ${ }^{7}$

Eine integrierte Standort- und Verkehrsplanung mit der Umsetzung des Leitbildes einer nutzungsgemischten, kompakten Stadt, der ,europäischen Stadt“ leistet sicher einen wesentlichen Beitrag zu lebendigen, lebenswerten und funktionsfähigen Städten sowie Regionen. Dies mag im innerörtlichen Verkehr auch zu Verkehrsverlagerungen oder einer geringfügigen Reduzierung der zurückgelegten Distanzen beitragen, die im Grundsatz erfreulich, angesichts der Höhe der notwendigen Reduzierung der $\mathrm{CO}_{2}-$ Emissionen aber unbedeutend sind. Hierzu müssen andere Ansätze und Akteure im Vordergrund stehen.

Eine relevante Reduzierung der $\mathrm{CO}_{2}$-Emissionen im Verkehrssektor ist vor allem die Aufgabe des Bundes und der EU, die zunächst im Zusammenhang mit dem Waldsterben seit den 1980er-Jahren und dann fortgesetzt gezeigt haben, wie sich Verkehrsemissionen erfolgreich reduzieren lassen: Durch wirksame Grenzwerte für die Fahrzeughersteller (trotz inzwischen offenbarer betrügerischer Machenschaften bei der Abgasmessung). Einen ergänzenden Schritt könnten verbindliche $\mathrm{CO}_{2}$-Kompensationszahlungen im Personen- und Güterverkehr für alle Verkehrsträger bilden. Die Einnahmen aus den Kompensationszahlungen sind dann in anderen Bereichen für die wirksame Reduzierung der $\mathrm{CO}_{2}$-Emissionen in gleichem Umfang zu nutzen. Die Höhe der Abgaben ergibt sich aus den für die Reduzierung erforderlichen Aufwendungen und steigt, je weiter dieses Potenzial ausgeschöpft ist.

Danksagung Die Analysen, die diesem Aufsatz zugrunde liegen, stammen aus mehreren durch die Deutsche Forschungsgemeinschaft (DFG) geförderten Vorhaben, insbesondere: Alltagsverkehr und Fernreisen (HO 3262/6-1), Alltag im Wandel des Geschlechterverhältnisses (HO 3262/4-2) und Veränderungen der Mobilität im Lebensverlauf (HO 3262/8-1). Wir danken der DFG für die Förderung. Gleichzei-

\footnotetext{
${ }^{7}$ Die Wirkungen deutlich erhöhter Kraftstoffsteuern (oder anderer Kostenanlastungen) können vielgestaltig sein und sind unter anderem abhängig von der konkreten Verwendung der zusätzlich vereinnahmten Mittel. Verhaltensanpassungen müssen sich nicht auf die Verkehrsnachfrage beschränken. Durchaus gewünscht können sie die Durchsetzung kraftstoffsparsamer Fahrzeugtechnik fördern. Sie können aber auch zur Reduzierung von Ausgaben außerhalb des Verkehrsbereichs (z. B. billigeres Einkaufen, Verzicht auf private Altersvorsorge) oder ebenfalls abhängig von der Mittelverwendung auch zu Veränderungen der Wirtschaftsleistung führen. Die Abschätzung auf das Verkehrsverhalten und die verkehrsbedingten $\mathrm{CO}_{2}$-Emissionen sind sehr komplex und unserer Ansicht nach gerade bei starken Anhebungen nicht zuverlässig vorhersehbar. In der Gesamteinschätzung sehen wir aber als wichtigere Barriere die fehlende und nicht absehbare gesellschaftliche und politische Akzeptanz einer solchen Maßnahme.
} 
tig hat der Beitrag von intensiven und kontroversen Diskussionen innerhalb des Arbeitskreises „Wechselwirkungen von Mobilität und Raumentwicklung im Kontext des gesellschaftlichen Wandels" der Akademie für Raumforschung und Landesplanung (ARL) profitiert. Wir danken den weiteren Mitgliedern des Arbeitskreises Janna Albrecht, Prof. Dr. Klaus J. Beckmann, Prof. Dr. Jens S. Dangschat, Prof. Dr. Carsten Gertz, Tanja Göbler, Dr. Martina Hülz, Georg-Friedrich Koppen, Prof. Dr. Bert Leerkamp, Prof. Dr. Ulrike Reutter, Christoph Scheck, Dr. Dirk Wittowksy für die anregenden Diskussionen, den Widerspruch, bisweilen auch für die Zustimmung, und der ARL für die Unterstützung.

\section{Literatur}

Aamaas, B.; Borken-Kleefeld, J.; Peters, G. P. (2013): The climate impact of travel behavior: A German case study with illustrative mitigation options. In: Environmental Science \& Policy 33, 11, 273-282.

Axhausen, K.W. (2007): Activity spaces, biographies, social networks and their welfare gains and externalities: some hypotheses and empirical results. In: Mobilities 2, 1, 15-36.

Bauer, U.; Holz-Rau, C.; Scheiner, J. (2005): Standortpräferenzen, intraregionale Wanderungen und Verkehrsverhalten. In: Raumforschung und Raumordnung 63, 4, 266-278.

Beckmann, K. J.; Holz-Rau, C.; Rindsfüser, G.; Scheiner, J. (2005): Mobilität älterer Menschen - Analysen und verkehrsplanerische Konsequenzen. In: Echterhoff, W. (Hrsg.): Strategien zur Sicherung der Mobilität älterer Menschen. Köln, 43-71.

Blossfeld, H.-P.; Timm, A. (Hrsg.) (2003): Who marries whom? Educational systems as marriage markets in modern societies. A comparison of thirteen countries. Dordrecht. $=$ European Studies of Population 12.

BMVI - Bundesministerium für Verkehr und digitale Infrastruktur (Hrsg.) (2014): Verkehr in Zahlen 2014/2015. Hamburg.

Boarnet, M. G. (2011): A Broader Context for Land Use and Travel Behavior, and a Research Agenda. In: Journal of the American Planning Association 77, 3, 197-213.

Canzler, W.; Kaufmann, V.; Kesselring, S. (Hrsg.) (2008): Tracing Mobilities. Aldershot.

Cao, J. (2014): Residential self-selection in the relationships between the built environment and travel behavior. In: Journal of Transport and Land Use 7, 3, 1-3.

Castells, Manuel (2004): Das Informationszeitalter. 3 Bände. Opladen.

Cervero, R. (2003): Road Expansion, Urban Growth, and Induced Travel: A Path Analysis. In: Journal of the American Planning Association $69,2,145-163$.

Chatman, D.G. (2014): Estimating the effect of land use and transportation planning on travel patterns: Three problems in controlling for residential self-selection. In: Journal of Transport and Land Use 7, 3, 47-56.

Dargay, J. (2007): The effect of prices and income on car travel in the UK. In: Transportation Research Part A 41, 10, 949-960.

Einig, K.; Pütz, T. (2007): Regionale Dynamik der Pendlergesellschaft: Entwicklung von Verflechtungsmustern und Pendeldistanzen. In: Informationen zur Raumentwicklung 2/3, 73-91.

Elldér, E. (2014): Residential location and daily travel distances: the influence of trip purpose. In: Journal of Transport Geography 34, $1,121-130$.

Ewing, R.; Cervero, R. (2010): Travel and the built environment: A meta-analysis. In: Journal of the American Planning Association 76, 3, 265-294.

Frändberg, L. (2009): How normal is travelling abroad? Differences in transnational mobility between groups of young Swedes. In: Environment and Planning A 41, 3, 649-667.

Frändberg, L.; Vilhelmson, B. (2003): Personal mobility: a corporeal dimension of transnationalisation. The case of long-distance travel from Sweden. In: Environment and Planning A 35, 10, 1751-1768.

Frändberg, L.; Vilhelmson, B. (2011): More or less travel: personal mobility trends in the Swedish population focusing gender and cohort. In: Journal of Transport Geography 19, 6, 1235-1244.

Frank, S. (2013): Innere Suburbanisierung? Mittelschichteltern in den neuen innerstädtischen Familienenklaven. In: Kronauer, M.; Siebel, W. (Hrsg.): Polarisierte Städte. Soziale Ungleichheit als Herausforderung für die Stadtpolitik. Frankfurt, New York, 69-89.

Frei, A.; Axhausen, K.W.; Ohnmacht, T. (2009): Mobilities and Social Network Geography: Size and Spatial Dispersion - the Zurich Case Study. In: Ohnmacht, T.; Maksim, H.; Bergman, M. M. (Hrsg.): Mobilities and Inequality. Burlington, 99-120.

Geier, S.; Holz-Rau, C.; Krafft-Neuhäuser, H. (2001): Randwanderung und Verkehr. In: Internationales Verkehrswesen, 53, 1-2, 22-26.

Gertz, C.; Holz-Rau, C.; Rau, P. (1994): Verkehrsvermeidung durch Raumstruktur - Personenverkehr. Studienprogramm der Enquete-Kommission Schutz der Erdatmosphäre, Band 4, Teilband 1. Bonn.

Guth, D.; Siedentop, S.; Holz-Rau, C. (2012): Erzwungenes oder exzessives Pendeln? Zum Einfluss der Siedlungsstruktur auf den Berufspendelverkehr. In: Raumforschung und Raumordnung 70, 6, 485-499.

Handy, S. (1992): Regional versus Local Accessibility: Neo-Traditional Development and its Implications for Non-Work Travel. In: Built Environment 18, 4, 253-267.

Hard, G. (2008): Der Spatial Turn, von der Geographie her beobachtet. In: Döring, J.; Thielmann, T. (Hrsg.): Spatial Turn. Das Raumparadigma in den Kultur- und Sozialwissenschaften. Münster, 263-315.

Heinze, G. W. (1979): Verkehr schafft Verkehr. Ansätze zu einer Theorie des Verkehrswachstums als Selbstinduktion. In: Berichte zur Raumforschung und Raumplanung 23, 4/5, 9-32.

Herfert, G.; Osterhage, F. (2012): Wohnen in der Stadt: Gibt es eine Trendwende zur Reurbanisierung? Ein quantitativ-analytischer Ansatz. In: Brake, K.; Herfert, G. (Hrsg.): Reurbanisierung. Materialität und Diskurs in Deutschland. Wiesbaden, 86-112.

Hesse, M.; Scheiner, J. (2007): Räumliche Mobilität im Kontext des sozialen Wandels: Eine Typologie multilokalen Wohnens. In: Geographische Zeitschrift 95, 3, 138-154.

Hesse, M.; Scheiner, J. (2010): Mobilität, Erreichbarkeit und gesellschaftliche Teilhabe: Die Rolle von strukturellen Rahmenbedingungen und subjektiven Präferenzen. In: Vierteljahreshefte zur Wirtschaftsforschung 79, 2, 94-112.

HIS - Institut für Hochschulforschung; DAAD - Deutscher Akademischer Austauschdienst (2013): Ausgewählte Ergebnisse der 4. Befragung deutscher Studierender zu studienbezogenen Aufenthalten in anderen Ländern 2013. Berlin.

Holden, E.; Linnerud, K. (2011): Troublesome Leisure Travel: The Contradictions of Three Sustainable Transport Policies. In: Urban Studies 48, 14, 3087-3106.

Holz-Rau, C. (1997): Siedlungsstrukturen und Verkehr. Bonn. = Materialien zur Raumentwicklung 84.

Holz-Rau, C.; Fromberg, A.; Gwiasda, P.; Rau, P.; Scheiner, J.; Trubbach, K. (1999): Nutzungsmischung und Stadt der kurzen Wege. Werden die Vorzüge einer baulichen Mischung im Alltag genutzt? Bonn. = Werkstatt: Praxis 7/1999.

Holz-Rau, C.; Scheiner, J.; Sicks, K. (2014): Travel distances in daily travel and long-distance travel: What role is played by urban form? In: Environment and Planning A 46, 2, 488-507.

Jenks, M.; Burton, E.; Williams, K. (Hrsg.) (1997): The compact city: A sustainable urban form? London.

Kabisch, S.; Steinführer, A.; Haase, A. (2012): Reurbanisierung aus soziodemographischer Perspektive: Haushalte und Quartierswandel in der inneren Stadt. In: Brake, K.; Herfert, G. (Hrsg.): Reurbanisierung. Materialität und Diskurs in Deutschland. Wiesbaden, $113-129$ 
Kagermeier, A. (1997): Siedlungsstruktur und Verkehrsmobilität. Eine empirische Untersuchung am Beispiel von Südbayern. Dortmund. $=$ Verkehr Spezial 3 .

Konrad, K. (2016): Mobiler Alltag im Wandel des Geschlechterverhältnisses. Wiesbaden. = Studien zur Mobilitäts- und Verkehrsforschung 29.

Kuhnimhof, T.; Armoogum, J.; Buehler, R.; Dargay, J.; Denstadli, J. M.; Yamamoto, T. (2012): Men Shape a Downward Trend in Car Use among Young Adults - Evidence from Six Industrialized Countries. In: Transport Reviews 32, 6, 761-779.

Kutter, E. (1975): Mobilität als Determinante städtischer Lebensqualität. In: Leutzbach, W. (Hrsg.): Verkehr in Ballungsräumen. Köln, 65-75. = Schriftenreihe der Deutschen Verkehrswissenschaftlichen Gesellschaft 24.

Lehner, F. (1963): Siedlung, Wohndichte und Verkehr. Bielefeld. = Schriftenreihe für Verkehr und Technik 17.

Lucas, K. (2012): Transport and social exclusion: where are we now? In: Transport Policy 20, 2, 105-113.

Metz, D. (2004): Travel time - variable or constant? In: Journal of Transport Economics and Policy 38, 3, 333-344.

Millard-Ball, A.; Schipper, L. (2011): Are We Reaching Peak Travel? Trends in Passenger Transport in Eight Industrialized Countries. In: Transport Reviews 31, 3, 357-378.

Mokhtarian, P. L.; Chen, C. (2004): TTB or not TTB, that is the Question: A Review and Analysis of the Empirical Literature on Travel Time (and Money) Budgets. In: Transportation Research A 38, 9-10, 643-675.

Motzkus, A. H. (2002): Dezentrale Konzentration - Leitbild für eine Region der kurzen Wege? Sankt Augustin. = Bonner Geographische Abhandlungen 107.

Næss, P. (2011): ,New urbanism“ or metropolitan-level centralization? A comparison of the influences of metropolitan-level and neighborhood-level urban form characteristics on travel behavior. In: Journal of Transport and Land Use 4, 1, 25-44.

Ottelin, J.; Heinonen, J.; Junnila, S. (2014): Greenhouse gas emissions from flying can offset the gain from reduced driving in dense urban areas. In: Journal of Transport Geography 41, 1-9.

Petrunoff, N.; Rissel, C.; Wen, L.M.; Martin, J. (2015): Carrots and sticks vs carrots: Comparing approaches to workplace travel plans using disincentives for driving and incentives for active travel. In: Journal of Transport \& Health 2, 4, 563-567.
Pütz, T. (2015): Verkehrsbild Deutschland. Pendlerströme. Quo navigant? Bonn. = BBSR-Analysen KOMPAKT 15/2015.

Rammler, S. (2001): Mobilität in der Moderne. Geschichte und Theorie der Verkehrssoziologie. Berlin.

Reichert, A.; Holz-Rau, C. (2014): Verkehrsmittelnutzung im Fernverkehr. In: Proff, H. (Hrsg.): Radikale Innovationen in der Mobilität. Wiesbaden, 429-444.

Scheiner, J. (2009): Sozialer Wandel, Raum und Mobilität. Empirische Untersuchungen zur Subjektivierung der Verkehrsnachfrage. Wiesbaden.

Scheiner, J. (2012): A century of motorisation in urban and rural contexts: paths of motorisation in German cities. In: Erdkunde 66, 4, 313-328.

Schmitz, S. (2001): Revolutionen der Erreichbarkeit. Gesellschaft, Raum und Verkehr im Wandel. Opladen.

Schwanen, T.; Dijst, M.; Dieleman, F. M. (2004): Policies for Urban Form and their Impact on Travel: The Netherlands Experience. In: Urban Studies 41, 3, 579-603.

Siedentop, S.; Stein, A.; Lanzendorf, M. (2005): Mobilität im suburbanen Raum. Neue verkehrliche und raumordnerische Implikationen des räumlichen Strukturwandels. Abschlussbericht. Dresden, Berlin, Erkner, Leipzig.

Sultana, S. (2005): Effects of Married-Couple Dual-Earner Households on Metropolitan Commuting: Evidence from the Atlanta Metropolitan Area. In: Urban Geography 26, 4, 328-352.

Surprenant-Legault, J.; Patterson, Z.; El-Geneidy, A. M. (2013): Commuting trade-offs and distance reduction in two-worker households. In: Transportation Research Part A 51, 5, 12-28.

Szalai, A. (Hrsg.) (1972): The Use of Time. Daily Activities of Urban and Suburban Populations in Twelve Countries. The Hague, Paris.

Taylor, B. D.; Morris, E.A. (2015): Public transportation objectives and rider demographics: are transit's priorities poor public policy? In: Transportation 42, 2, 347-367.

van Wee, B. (2015): Peak car: The first signs of a shift towards ICTbased activities replacing travel? A discussion paper. In: Transport Policy 42, 8, 1-3.

van Wee, B.; Handy, S. (2016): Key research themes on urban space, scale, and sustainable urban mobility. In: International Journal of Sustainable Transportation 10, 1, 18-24.

Wegener, M. (2009): Modelle der räumlichen Stadtentwicklung - alte und neue Herausforderungen. In: Stadt Region Land 87, 73-81. 